\title{
LA EDUCACIÓN EMOCIONAL DEL DOCENTE Y SU LABOR: UNA EXPERIENCIA DE APRENDIZAJE-SERVICIO EN LA UNIVERSIDAD Y SU EVALUACIÓN
}

\author{
Sarah Carrica-Ochoa \\ Universidad de Navarra
}

\begin{abstract}
RESUMEN: La Universidad posee la responsabilidad de formar profesionales, lo cual significa dotar a las personas de las competencias necesarias para enfrentarse al mundo con un compromiso con la justicia social. En este trabajo se muestra una experiencia de Aprendizaje-Servicio (ApS) -y su evaluacióncomo una manera de colaboración, servicio y mejora de la comunidad. En primer lugar, se analiza el contexto teórico del ApS como metodología en relación con el desarrollo comunitario y la inteligencia emocional. En segundo lugar, se describe una experiencia de ApS llevada a cabo en los grados de educación de la Universidad de Navarra. El objetivo ha sido acompañar al alumnado en el análisis de la actuación socioeducativa y su capacitación para intervenir pedagógicamente en ámbitos concretos. Por último, se presenta la evaluación realizada a través de cuestionarios de satisfacción y participación; así como una escala de análisis de capacidades trabajadas, realizada al efecto. Los resultados muestran que metodologías como el ApS logran un alto grado de satisfacción, compromiso y motivación del alumnado por la tarea.
\end{abstract}

PALABRAS CLAVE: Aprendizaje-Servicio, desarrollo comunitario, inteligencia emocional, capacidades.

\section{THE EMOTIONAL EDUCATION OF TEACHERS AND ITS TEACHING WORK: A EXPERIENCE OF SERVICE-LEARNING AT THE UNIVERSITY AND ITS EVALUATION}

\footnotetext{
ABSTRACT: University has the responsibility to train professionals, which means providing people with the necessary competences to face the world with an engagement to social justice. This paper presents a service-learning experience (SL) -and its evaluation- as a way of collaboration, service and improvement of community. Firstly, this work analyses SL and its theoretical context as a methodology in relation to community development and emotional
} 
intelligence. Secondly, a SL experience, conducted in grades of education at the University of Navarra, is described. The objective has been to accompany the students in the analysis of the socio-educational action and capacitate them to intervene pedagogically in specific areas. Finally, the evaluation made through satisfaction questionnaires is presented; also a scale analysis of professional skills worked, made to that effect. The results show that methodologies such as $\mathrm{SL}$ allow achieving a high degree of satisfaction, engagement and motivation of students for the task required.

KEYWORDS: Service-Learning, community development, emotional intelligence, skills.

Recibido: 21/03/2016

Aceptado: 17/07/2016

Correspondencia: Sarah Carrica-Ochoa, Universidad de Navarra, Facultad de Educación y Psicología, Campus Universitario, 31009 Pamplona, Navarra. Email: scarrica@unav.es.

\section{INTRODUCCIÓN}

Nuestro contexto en continuo cambio exige una formación de la ciudadanía que le dote de las herramientas necesarias para adaptarse (u enfrentarse) a dichos cambios y exigencias sociales. En este sentido, la función del sistema educativo es formar, de manera innovadora, una ciudadanía capaz de desarrollar al máximo sus habilidades y capacidades; de manera que reproduzca y asegure dicha innovación y transformación del mundo -en clave de justicia social- (Onu, Eskay, Obiyo, Igbo y Ezeanwu, 2012 , p. 230). En este sentido, las universidades poseen una gran responsabilidad en el empoderamiento de sus estudiantes a través de conocimientos, estrategias y actitudes específicas que les capaciten para vivir y trabajar en la sociedad que les corresponde (Campbell y Rozenyai, 2002).

Por otro lado, parece que el proceso de Bolonia (Bologna Working Group, 2005) aboga por un proyecto universitario centrado en el aprendizaje del estudiante en el cual se entremezclen la emoción, la conexión con la realidad, la interrelación de conocimientos y la colaboración entre personas; en aras de un aprendizaje profundo (Lantieri, 2009; Siegel, 2011). Todo lo cual supone un intento de desarrollar un modelo innovador centrado en el alumnado y su aprendizaje (Palomares, 2011). Y para ello es necesario replantear la enseñanza universitaria apostando por el uso de metodologías que incentiven la autonomía, pensamiento crítico y trabajo en equipo (Arandia y Fernández, 2012).

Precisamente, la metodología de Aprendizaje y Servicio (ApS en adelante) está orientada a lograr un proceso de enseñanza-aprendizaje activo que influya en el desarrollo individual (tanto personal como profesional) y social; añadiendo calidad, profundidad y extensión en la formación de los futuros profesionales (Martínez, 2010; Martínez, Martínez, Alonso y Gezuraga, 2013). De este modo se posibilita el desarrollo de unas competencias sociales y profesionales al mismo tiempo que se realizan 
actividades significativas, motivadoras, en contacto con un ámbito real y aprendiendo haciendo y en colaboración con otras personas y la comunidad (Alonso, Arandia, Martínez, Martínez y Gezuraga, 2013). Lo que además, facilitará el acercamiento y conocimiento mutuo, la creatividad, la expresión de emociones y la comprensión de la realidad desde distintos puntos de vista; tal y como se ha percibido a través de la experiencia de ApS realizada que se presenta a continuación.

La ciudadanía está expuesta y maneja información de todas partes del mundo pero muchas veces sin una explicación previa sobre dicho lugar (Bourn y Brown, 2011). Estos efectos en la ciudadanía y juventud en particular, se traducen en unas necesidades educativas específicas, lo cual requiere a su vez docentes con unas capacidades, cualidades o competencias concretas. ¿Pero están nuestros docentes preparados para dicha responsabilidad? ¿Los planes formativos universitarios de los grados de educación y las actividades realizadas van en dicha línea? ¿Están los estudiantes preparados para ser agentes de transformación social al acabar sus estudios?

Todas estas cuestiones hacen referencia a la labor docente, entendida en este trabajo como calidad docente, la cual hace referencia a aquellas iniciativas que tratan de mejorar los conocimientos, competencias y habilidades -en ejercicio o formación- de los docentes (Castilla, 2011). Desde el marco del Espacio Europeo de Educación Superior (EEES) se insta a la mejora de la calidad de la docencia universitaria (Martín-Gutiérrez, Conde-Jiménez y Mayor-Ruiz, 2014). La mejora de la calidad implica un entramado de variables relacionadas entre si, siendo el profesorado uno de los ejes centrales (Valcárcel, 2003). Fundamentalmente, se destacan dos elementos relacionados con el profesorado: su formación y su desarrollo profesional.

En este artículo se presenta una experiencia de ApS como propuesta formativa de futuros educadores. Dicha experiencia facilita atender a la formación de los estudiantes -a través del aprendizaje significativo y experiencial- y a su desarrollo profesional, ya que los estudiantes se encargan de preparar y poner en marcha la actividad. A estos dos elementos propuestos por Valcárcel (2003) se ha querido añadir el desarroIlo personal, concretamente el desarrollo de la inteligencia emocional. Se considera que las emociones juegan un papel esencial en la toma de decisiones, las cuales a su vez están condicionadas por la presión social, aspecto en el que la emoción del miedo posee una gran relevancia.

\section{El desarrollo comunitario a través del aprendizaje-servicio}

Las propuestas de implicación de la ciudadanía en el desarrollo comunitario son innumerables y a nivel internacional se multiplican los proyectos elaborados para la potenciación de la presencia de la sociedad civil (Carrica-Ochoa, 2012; CONCORD, 2011; Hogg, 2011; Glennie, Straw y Wild, 2012; Brown, 2013). El ApS es una actividad compleja que integra el servicio a la comunidad con el aprendizaje educativo. Se trata de una propuesta innovadora, pero al mismo tiempo también es una propuesta que parte de elementos muy conocidos: el servicio a la comunidad y, por supuesto, la transmisión de conocimientos, habilidades y valores. La novedad no reside en cada una de las partes que lo componen, sino en vincular estrechamente servicio y aprendizaje en una sola actividad educativa bien articulada y coherente (Puig, Batlle, Carme, Palos, 2007). 
El ApS es un método de enseñanza-aprendizaje intencional y estructurado en el que se da la coexistencia de dos componentes clave: los objetivos de aprendizaje académico y los objetivos de servicio a la comunidad. Los proyectos de ApS otorgan también un papel relevante a la reflexión, que es lo que posibilita la conexión entre aprendizaje y servicio. Martínez-Odría (2005) destaca cinco características esenciales del ApS:

1. Protagonismo del alumno en el proceso de participación social y de aprendizaje académico.

2. Atención a una necesidad real de la comunidad en la que se inserta la labor del estudiante.

3. Conexión de la actividad de servicio comunitario con los objetivos curriculares de aprendizaje.

4. Realización de un proyecto de servicio (planificación, preparación, implementación y evaluación) que será presentado a la comunidad.

5. Reflexión (antes, durante y después del proyecto de servicio) y realización de conexiones con los aprendizajes curriculares.

Por otro lado, el desarrollo comunitario supone un esfuerzo social y educativo que busca mejorar las condiciones de vida de la comunidad y de la ciudadanía que en ella habita, sirviendo y ayudándose de los recursos intrínsecos. Tal esfuerzo social y educativo se traduce en actividades o procesos que realicen los siguientes pasos:

1) Estudio de la realidad,

2) Programación de actividades,

3) Acción social conducida de manera racional,

4) Evaluación de lo realizado (Ander-Egg, 2000).

En este sentido, el ApS puede convertirse en una excelente herramienta de desarrollo comunitario ya que, en cierta manera, sigue tales pasos (Ander-Egg, 2000). Además, el ApS, si bien es una metodología educativa, guarda una estrecha relación con otros campos más allá del ámbito educativo formal. Por ejemplo, el ApS funciona como herramienta de desarrollo comunitario, de cohesión de la comunidad, ayudando a conformar un proyecto integral de educación para la ciudadanía y de fortalecimiento de la sociedad. La razón fundamental es que a través del ApS se establece un puente entre lo académico y lo social, abordando la formación de ciudadanía y profesionales con ética y un amplio sentido de la participación social (Gil-Gómez, Moline-García, Chiva-Bartoll y García-López, 2014). Además, las iniciativas que tratan de mejorar el entorno proporcionan experiencias reales de inclusión a los participantes, contribuyendo a la cohesión social.

A través del ApS se incrementa, pues, el capital social, creando un efecto multiplicador. Diferentes estudios (Billing y Waterman, 2014; Bringe y Hatcher, 2009; Hérbet y Hauf, 2015; Battle, 2010) muestran los beneficios de este tipo de experiencias para la comunidad -tanto educativa como social- como la mejora de: la participación y compromiso de los jóvenes; el conocimiento mutuo por parte de los agentes sociales y se creación de lazos de comunidad; la eficacia de las actuaciones de cada agente 
social; el nivel cultural de la población; la autoimagen, autoestima y confianza; la comunicación entre los diferentes sectores de población; la capacidad de la población para enfrentarse a retos y a adversidades; o la responsabilidad ciudadana.

Es decir, las experiencias de ApS facilitan las condiciones para el desarrollo de capacidades cognitivas y emotivas gracias al contacto con la vida real, lugar donde los contenidos aprendidos se comparten y completan. De modo que el ApS permite realizar aprendizajes más cercanos a la práctica profesional (Alonso, 2004), al mismo tiempo que conectan con la dimensión emocional y social de la persona.

\section{El desarrollo emocional, la labor docente y el aprendizaje-servicio}

El mundo moderno globalizado y diverso requiere de unas personas que trabajen y desarrollen no solo su capacidad cognitiva sino también la social y emocional. Esta inteligencia emocional tiene que ver con la manera de verse a sí mismo, con las conductas autodeterminadas de las personas, con la actitud positiva y resiliente y como no, con la manera de relacionarse con el resto de personas que componen la sociedad. Es ahí, en ese punto, donde se relaciona inteligencia emocional con desarrollo comunitario, contando con el ApS como herramienta facilitadora y mediadora entre tales objetivos: el desarrollo personal y social; de una forma participativa, inclusiva y justa.

Del mismo modo que Goleman habla de "trabajadores estrella" (Goleman, 1998) se podría hablar de "ciudadanía estrella", formada por aquellas personas que son capaces de establecer conexiones sociales con otros ámbitos de su comunidad bien sea para resolver problemas, mejorar situaciones o incluso responder a necesidades de manera conjunta.

Las experiencias personales y nuestra manera de funcionar en sociedad tienen un reflejo en nuestra dimensión profesional y viceversa. En este sentido Gewerc (2011) apunta que la construcción de la identidad profesional docente comienza a definirse en el transcurso de la socialización primaria y continúa en el proceso de formación inicial con un mayor impacto que en otros momentos de la trayectoria profesional. Como resultado, se construyen un conjunto de competencias, cuya interiorización va a identificar al docente como una persona conformada por múltiples experiencias de vida (Martín-Gutiérrez, Conde-Jiménez y Mayor-Ruiz, 2014). Esto definirá al docente y su labor haciéndole en cierta manera, único e irrepetible, atendiendo a sus propios rasgos de personalidad, motivaciones y las actitudes como docente.

Por ello, para la construcción social del docente, es decir, el desarrollo de su competencia social, es crucial la conexión con la comunidad y contexto que le rodea (Zabalza, 2011). Esto exige a la Universidad asumir el compromiso de establecer metodologías que aborden y fomenten el desarrollo de la dimensión social de los docentes (Debowsky, 2012). Lo cual a su vez, implica incluir la inteligencia emocional como otro de los aprendizajes básicos de los futuros docentes. En las relaciones con los demás, en nuestro trabajo y por supuesto en la labor docente, influyen nuestras emociones. Según un estudio de Mayor (2011) la ansiedad, incertidumbre y miedo que sienten los docentes en su labor disminuye cuando es compartida con compañeros. En este sentido resulta interesante proponer espacios en los que se pueda reflexionar sobre los propios miedos.

Ante todo esto, los proyectos de ApS ayudan a construir espacios y experiencias en las que, por un lado poner en práctica la labor docente ya que se crean situacio- 
nes reales en las que intervenir. Esta conexión con la realidad, además de cumplir una labor de servicio con la comunidad, facilita el aprendizaje colaborativo y por lo tanto el desarrollo de la competencia social. Por otro lado, el ApS, dada su condición experiencial, facilita poner en juego una serie de emociones y habilidades personales que a su vez revertirán en el desarrollo de la competencia social y labor docente.

En conclusión, los proyectos de ApS guardan una estrecha relación con el desarrollo emocional, social y profesional, ya que consiste en poner en práctica la futura profesión con personas y situaciones reales de la comunidad. Por lo tanto supone una excelente oportunidad de experimentar la futura labor docente al mismo tiempo que se trabajan cuestiones personales que ya se ha visto que poseen su influencia en lo profesional.

\section{UNA EXPERIENCIA DE APRENDIZAJE-SERVICIO EN LA UNIVERSIDAD}

El presente proyecto de ApS se desarrolla en la Facultad de Educación y Psicología de la Universidad de Navarra durante el primer semestre del curso académico 20152016. Sin embargo, fomentando el conocimiento mutuo, la participación y servicio a la comunidad; en la actividad intervinieron otras dos instituciones de la Comunidad Foral de Navarra: la Fundación Ilundain Haritz-Berri y el Museo de la Universidad de Navarra. Concretamente la actividad surge dentro de una asignatura "Fundamentos y estrategias de la actuación socioeducativa I" impartida en los grados de educación (27 alumnos en total). Fue en el marco de dicha asignatura donde se planteó trabajar conjuntamente con un colectivo en riesgo de exclusión social de la comarca de Pamplona: 15 alumnos que se encuentran en el Programa de Currículum Adaptado (PCA en adelante) en la Fundación Ilundain Haritz-Berri.

\section{Estructura de la asignatura marco de la experiencia de ApS}

La asignatura Fundamentos y estrategias de actuación socioeducativa está dividida en dos partes: I y II; impartidas en dos semestres diferentes. La intención de tal asignatura es acompañar al alumnado en el análisis de la actuación socioeducativa y su capacitación para intervenir pedagógicamente en ámbitos concretos. Para lo cual en la primera parte de la asignatura (primer semestre) se plantea el estudio sobre temas como el desarrollo humano, social y comunitario; la participación ciudadana; el entorno y sus características; o los elementos de la intervención socioeducativa. En el segundo semestre y como complemento, la segunda parte se centra en los entornos y realidades socioculturales, tales como la educación patrimonial, o la educación para el ocio y el tiempo libre.

\section{Objetivos de la asignatura}

Los objetivos planteados de la asignatura son principalmente, que el alumnado logre:

1. Planificar una actividad de Desarrollo Comunitario.

1.1. Conocer todas las fases y requisitos del desarrollo comunitario. 
2. Realizar una actividad de Aprendizaje-Servicio.

2.1. Conocer la Fundación Ilundain y su alumnado.

2.2. Reflexionar sobre la labor docente identificando cualidades positivas y negativas de la figura del educador/a a través de la experiencia personal vivida en el sistema educativo.

2.3. Identificar miedos personales y trabajar sobre las distintas maneras de afrontarlos.

3. Desarrollar las siguientes capacidades: compromiso ético, planificación y gestión del tiempo, motivación, apreciación de la diversidad, relación con los demás, aplicación de conocimientos.

3.1. Gestionar el propio trabajo dentro del grupo de trabajo e inter-grupos.

Estos objetivos van a implicar el desarrollo de una serie de conocimientos (saber) destrezas (saber hacer) y actitudes (saber ser) en el alumnado, las cuales se detallan en el Figura 1.

\begin{tabular}{|c|c|c|}
\hline Conocimientos (saber) & Destrezas (saber hacer) & Actitudes (saber ser) \\
\hline $\begin{array}{l}\text { Diferencias las etapas } \\
\text { del desarrollo humano } \\
\text { (moral, emocional, } \\
\text { cognitivo, biológico, } \\
\text { social) significativas para } \\
\text { la intervención educativa. } \\
\text { Diferentes modelos } \\
\text { de intervención } \\
\text { socioeducativa. }\end{array}$ & $\begin{array}{c}\text { Tomar decisiones educativas } \\
\text { de acuerdo con las diferencias } \\
\text { individuales y sociales para escoger } \\
\text { el modelo educativo más adecuado } \\
\text { en cada situación. } \\
\text { Capacidad toma de decisiones. } \\
\text { Capacidad de planificación. } \\
\text { Capacidad de organización. } \\
\text { Capacidad de coordinación. } \\
\text { Contribuir al establecimiento } \\
\text { de políticas o estrategias de } \\
\text { actuación para la integración, la } \\
\text { interculturalidad y la equidad. }\end{array}$ & $\begin{array}{c}\text { Respeto a las diferencias } \\
\text { individuales y culturales. } \\
\text { Tolerancia. } \\
\text { Equidad. } \\
\text { Capacidad crítica. }\end{array}$ \\
\hline
\end{tabular}

Figura 1. Conocimientos, destrezas y actitudes de la asignatura

\section{Temática de la experiencia de ApS}

La experiencia de ApS gira en torno a dos grandes temas escogidos la educación emocional y la labor docente. La pertinencia de unir estas cuestiones se debe a que el ApS, como metodología, proporciona una serie de características favorables para trabajar la educación emocional: aprendizaje experiencial, protagonismo del alumnado en la realización del trabajo, la búsqueda de cambios en el entorno, entre otras. Todo ello, sirviendo como base idónea además para la reflexión sobre las cualidades del docente a través del ejercicio y puesta en práctica de ciertas funciones correspondientes a dicha labor. 


\section{Metodología}

La asignatura ha sido dividida en dos grandes bloques. En una primera fase se trataba de capacitar al alumnado para que pudiera tener las herramientas suficientes y adecuadas para la realización del proyecto de ApS dentro de un marco de desarrollo comunitario. De modo que, en la segunda parte, las clases se convirtieron en lugares de trabajo en equipos.

Las fases o momentos seguidos para la intervención han sido: 1) Sensibilización y motivación (a través de las clases teóricas), 2) Selección del ámbito de intervención, 3) Capacitación para la intervención, 4) Promoción de la organización y puesta en marcha de actividades socioeducativas.

Teniendo asumido como punto de partida que el ApS, como metodología requiere identificar en el entorno próximo una necesidad social y responder a ella mediante una acción para mejorar esa realidad, surgieron las primeras preguntas que dieron lugar a la actividad que aquí se presenta. ¿Qué podemos hacer? ¿Estamos capacitados para aportar algo a alguien de verdad? ¿Cómo lo haremos? ¿A quién/quiénes?

Una vez escogida la Fundación Ilundain Haritz-Berri y tras una visita que sirvió de contextualización y toma de contacto con la realidad, comenzó el trabajo por equipos en clase. Se propuso a los estudiantes escoger en qué equipo participar según cuáles fueran sus puntos fuertes. La relación de los equipos y el encargo de cada uno se resumen en la Figura 2.

\section{Equipo}

\section{Función}

\begin{tabular}{ll}
\hline a) Fundamentación teórica & $\begin{array}{l}\text { Documentación sobre la metodología del ApS y su relación } \\
\text { con la intervención socioeducativa }\end{array}$ \\
\hline b) Perfil psicopedagógico & $\begin{array}{l}\text { Conocer a fondo la fundación y el perfil de alumnado con } \\
\text { el que trabajan. Entrevistar al coordinador del Programa de } \\
\text { Currículo Adaptado (PCA) }\end{array}$ \\
\hline C) Programación & $\begin{array}{l}\text { Seguimiento del trabajo del resto de grupos asegurándose de } \\
\text { que se cumplen todas las partes del diseño de la intervención } \\
\text { socioeducativa, así como la elaboración de una memoria. }\end{array}$ \\
d) Creatividad & $\begin{array}{l}\text { Búsqueda de las actividades a realizar de manera que sean } \\
\text { creativas e innovadoras. Entrevista con el responsable de } \\
\text { actividades educativas del Museo Universidad de Navarra } \\
\text { Comunicación externa: creación de un blog, un slogan de } \\
\text { la actividad, así como redacción de la nota de prensa final. }\end{array}$ \\
\hline
\end{tabular}

Figura 2. Relación de equipos y sus funciones

\section{Evaluación de la asignatura}

La evaluación de la asignatura se estructura de la siguiente manera. Se evalúa la capacidad mostrada por el alumnado en distintas tareas, las cuales, con sus puntuaciones correspondientes, son:

a) Capacidad de participación tanto en el contexto del aula como el día de la experiencia: 1 punto evaluado por observación. 
b) Capacidad de aplicación del contenido aprendido: 4 puntos evaluados a través de la corrección de una memoria final realizada por todos los equipos.

c) Capacidad de diagnóstico y evaluación: 2 puntos a través de la presentación de una memoria del trabajo realizado por equipos.

d) Capacidad de reflexión: 3 puntos evaluados por medio de una reflexión individual.

\section{EVALUACIÓN DE UNA EXPERIENCIA DE APRENDIZAJE-SERVICIO}

El presente apartado muestra una propuesta de evaluación de experiencias de ApS. En este sentido se ha buscado que el alumnado evaluara una serie de capacidades así como su satisfacción con la experiencia.

\section{Muestra}

27 alumnos universitarios de la asignatura "Fundamentos y estrategias de la actuación socioeducativa I" impartida en los grados de $4^{\circ}$ de Pedagogía (6), 4º Pedagogía con Magisterio Infantil (11) y $5^{\circ}$ de Pedagogía con Magisterio Primaria (10).

\section{Objetivos e hipótesis}

Objetivo general

El objetivo general de la investigación es comprender la opinión del alumnado respecto a la experiencia de ApS sobre cuestiones a nivel personal y grupal.

\section{Objetivos específicos}

1. Conocer la satisfacción del alumnado en su trabajo por equipos, así como el realizado por el grupo-clase.

2. Comprobar si el alumnado reconoce haber desarrollados aspectos de las capacidades de: compromiso ético, planificación y gestión del tiempo, motivación, apreciación de la diversidad, relación con los demás, aplicación de conocimientos.

A cada uno de los objetivos específicos le corresponde una hipótesis:

H1. La experiencia de ApS proporcionará un grado de satisfacción alto con la tarea realizada.

H2. Los estudiantes valorarán de manera positiva su adquisición y desarrollo de capacidades (personales y profesionales) a través de la experiencia.

\section{Instrumentos}

Con el fin de conocer la opinión del alumnado ante experiencias educativas como las que facilita la metodología del ApS, se aplicaron tres cuestionarios creados "ad hoc" los cuales se detallan en el cuadro 3 junto con la relación de objetivos de cada uno de ellos. 


\begin{tabular}{ll}
\hline Técnica & Objetivo \\
\hline Autoevaluación de capacidades & $\begin{array}{l}\text { Identificar el nivel en que los estudiantes consideran } \\
\text { haber adquirido las capacidades genéricas y específicas } \\
\text { durante la aplicación del proyecto de ApS. } \\
\text { Evaluar el trabajo realizado por los miembros del } \\
\text { equipostionario de evaluación } \\
\begin{array}{l}\text { trabajo en equipo } \\
\text { Cuestionario de evaluación } \\
\text { trabajo grupo-clase }\end{array}\end{array} \quad \begin{array}{l}\text { Evaluar el trabajo de coordinación realizado entre } \\
\text { equipos. }\end{array}$ \\
\hline
\end{tabular}

Figura 3. Relación de objetivos e instrumentos del estudio

Los tres cuestionarios contestados por el alumnado al término del proyecto de ApS son: Cuestionario de evaluación del trabajo por equipos (Figura 4); Cuestionario de evaluación trabajo grupo-clase (Figura 5); y Cuestionario de autoevaluación de las capacidades trabajadas. Este último es una adaptación del cuestionario de MartínezVivot y Folgueiras (2015). El Cuadro 6 muestra la relación de las capacidades evaluadas y sus indicadores-ítems.

1. Todos los miembros del grupo hemos colaborado

2. El trabajo realizado era el pedido por la profesora

3. He cumplido con mi parte en el grupo

4. La distribución del trabajo entre los miembros del grupo ha sido equitativo

Figura 4. Ítems del Cuestionario de evaluación trabajo en equipo

1. Todos los miembros de la clase hemos colaborado.

2. El trabajo realizado era el pedido.

3. La colaboración entre los grupos ha sido buena.

4. El esquema seguido en la programación estaba bien organizado.

5. Hemos alcanzado los objetivos propuestos.

6. Las actividades han sido desarrolladas correctamente.

7. Nos hemos ajustado bien a los tiempos.

8. El clima de trabajo ha sido bueno.

9. Satisfacción final con el proyecto.

Figura 5. Ítems del Cuestionario de evaluación trabajo grupo-clase

\begin{tabular}{lc}
\hline Ítem indicador de la capacidad & Capacidades \\
\hline 1. Me he sentido responsable de mi trabajo. & Compromiso ético \\
2. Me he esforzado todo lo que he podido en las acciones. & \\
3. Considero que haber participado activamente en las & \\
actividades del proyecto me ha hecho crecer como persona. & \\
4. Comprendo la relación que hay entre el servicio y el aprendizaje. & \\
5. He tenido una excelente asistencia y si he faltado he avisado. & $\begin{array}{l}\text { Planificación y gestión } \\
\text { 6. No he podido dedicar todo el tiempo que hubiese querido a }\end{array}$ \\
la asignatura. & \\
7. He llegado siempre puntual. &
\end{tabular}


8. He estado muy motivada/o para realizar las actividades

9. He participado activamente en las actividades

10. Me he esforzado por conocer y comprender personas de un contexto diferente al que estoy acostumbrada/o.

(Auto)Motivación

1. Hemos fortalecido lazos con la comunidad.

12. La relación con el grupo-clase ha sido buena.

13. He notado un buen clima de clase.

14. Considero que haber participado activamente en las actividades del proyecto me ha dado herramientas para mi futura profesión.

Apreciación de la diversidad

Capacidad de relación

Aplicación conocimientos

Figura 6. Relación de las capacidades evaluadas y sus indicadores

\section{Resultados}

\section{Cuestionario de evaluación del trabajo en equipo}

El 100\% del alumnado escogió las opciones bastante y mucho (4 y 5) para valorar su satisfacción tanto con su labor en el equipo como el realizado por el equipo. Es decir, los ítems 2 y 3: "el trabajo realizado era el pedido por la profesora" y "he cumplido con mi parte en el grupo" son los que han recibido una mayor puntuación con unas medias de 4,8 y 4,7 respectivamente.

Por otro lado, los ítems relacionados con la equidad de la repartición de las tareas entre los miembros del equipo (ítem 4) así como con la colaboración entre los mismos (ítem 1) recibieron unas medias más bajas, de 4,3 donde cuyas puntuaciones están además más dispersas tan y como puede apreciarse en el Figura 7.

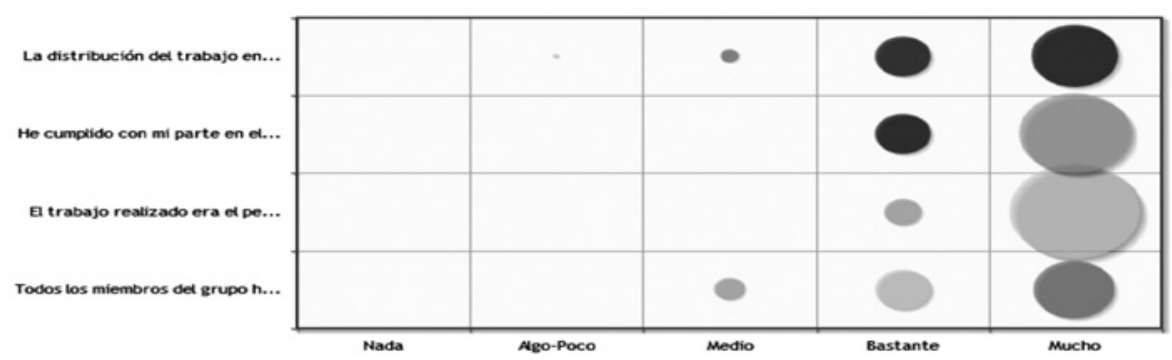

Figura 7. Satisfacción del trabajo en equipo

\section{Cuestionario de evaluación del trabajo grupo-clase}

En la tabla 1 se muestran los resultados de la satisfacción con el trabajo del grupo clase. El 100\% del alumnado ha escogido "bastante" o "todo", es decir, las puntuaciones 4 o 5, en los ítems 2 y 9, obteniendo unas medias de 4,8 en ambos casos. Lo cual supone una coherencia en las respuestas ya que en el ítem 2 se pregunta acerca de si el resultado final era el pedido por la docente (es decir, satisfacción con el resultado) y en el ítem 9 se preguntaba expresamente por la satisfacción final con el proyecto. 


\begin{tabular}{lc}
\hline Ítems & Puntuación media (M) \\
\hline 1. Todos los miembros de la clase hemos colaborado. & 4,3 \\
\hline 2. El trabajo realizado era el pedido. & 4,8 \\
3. La colaboración entre los grupos ha sido buena. & 4,4 \\
4. El esquema seguido en la programación estaba bien organizado. & 4,4 \\
5. Hemos alcanzado los objetivos propuestos. & 4,7 \\
6. Las actividades han sido desarrolladas correctamente. & 4,7 \\
7. Nos hemos ajustado bien a los tiempos. & 4,3 \\
8. El clima de trabajo ha sido bueno. & 4,6 \\
\hline 9. Satisfacción final con el proyecto. & 4,8 \\
\hline
\end{tabular}

Figura 8. Cuestionario sobre la valoración del trabajo global entre equipos

En general la valoración del trabajo del grupo-clase es alta, todas las puntuaciones medias son mayores de 4, siendo superior en los ítems que hacen referencia a la satisfacción final y general (ítems 2 y 9 con unas medias de 4,8), a los objetivos alcanzados (ítem 5 con 4,7 de media) y a su adecuación con las actividades las cuales, a su vez, se han desarrollado adecuadamente (4,7 de media para el ítem 6).

Sin embargo, reciben peor puntuación los ítems que tienen que ver con la colaboración equitativa de los miembros de la clase (ítem 1=4,3), el acomodamiento a los tiempos (ítem 7=4,3), la estructura del trabajo (ítem $4=4,4$ ), e incluso trabajo colaborativo (ítem $3=4,4)$. Es decir, la organización del trabajo entre grupos ha sido uno de los puntos peor valorados, lo cual coincide con los resultados anteriores obtenidos de la evaluación por equipos. Pese a la leve disminución en las puntuaciones del trabajo colaborativo, el clima de trabajo se puntúa con un 4,6 (ítem 8).

\section{Capacidades trabajadas}

En cuanto a las capacidades trabajadas, el alumnado expresa, en general, un nivel de satisfacción alto (Figura 9) con las dimensiones desarrolladas. Atendiendo a los resultados obtenidos (Figura 10: donde se muestran las puntuaciones medias obtenidas en cada ítem junto a su desviación típica) el alumnado realiza una sobresaliente valoración de las capacidades trabajadas gracias a la experiencia de ApS vivida.

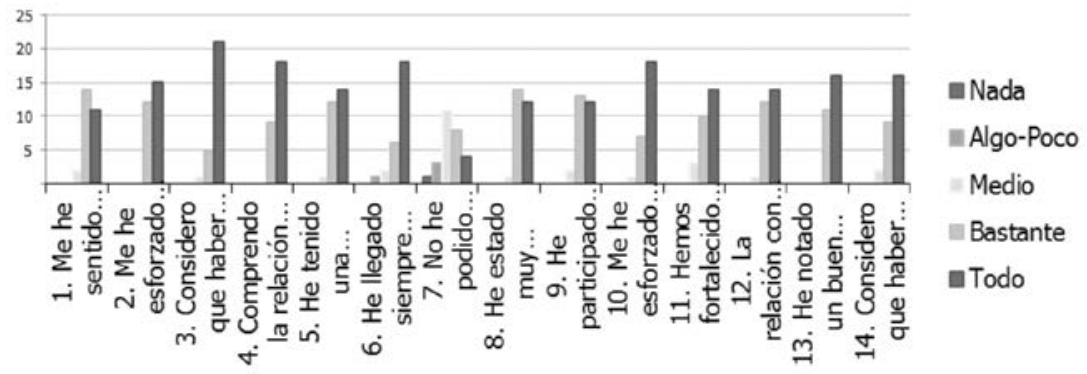

Figura 9. Opinión alumnado capacidades trabajadas 


\begin{tabular}{|c|c|c|c|}
\hline Ítem & $\begin{array}{l}\text { Media } \\
(\mathrm{M})\end{array}$ & $\begin{array}{l}\text { Desviación } \\
\text { típica (DT) }\end{array}$ & $\begin{array}{l}\text { Bastante- } \\
\text { Todo }\end{array}$ \\
\hline 1. Me he sentido responsable de mi trabajo. & 4 & 1 & $93 \%$ \\
\hline 2. Me he esforzado todo lo que he podido en las acciones. & 5 & 1 & $100 \%$ \\
\hline $\begin{array}{l}\text { 3. Considero que haber participado activamente en } \\
\text { las actividades del proyecto me ha hecho crecer como } \\
\text { persona. }\end{array}$ & 5 & 1 & $100 \%$ \\
\hline $\begin{array}{l}\text { 4. Comprendo la relación que hay entre el servicio y el } \\
\text { aprendizaje. }\end{array}$ & 5 & 0,4804 & $100 \%$ \\
\hline $\begin{array}{l}\text { 5. He tenido una excelente asistencia y si he faltado he } \\
\text { avisado. }\end{array}$ & 4,4815 & 0,5798 & $96 \%$ \\
\hline 6. He llegado siempre puntual. & 4,5185 & 0,8024 & $89 \%$ \\
\hline $\begin{array}{l}\text { 7. No he podido dedicar todo el tiempo que hubiese } \\
\text { querido a la asignatura. }\end{array}$ & 3,4074 & 1,0099 & $70 \%$ \\
\hline $\begin{array}{l}\text { 8. He estado muy motivada/o para realizar las } \\
\text { actividades. }\end{array}$ & 4,4074 & 0,5724 & $96 \%$ \\
\hline 9. He participado activamente en las actividades. & 4,3704 & 0,6293 & $93 \%$ \\
\hline $\begin{array}{l}\text { 10. Me he esforzado por conocer y comprender personas } \\
\text { de un contexto diferente al que no estoy acostumbrada/o. }\end{array}$ & 4,6538 & 0,5616 & $93 \%$ \\
\hline 11. Hemos fortalecido lazos con la comunidad. & 4,4074 & 0,6939 & $89 \%$ \\
\hline 12. La relación con el grupo-clase ha sido buena. & 4,4815 & 0,5798 & $96 \%$ \\
\hline 13. He notado un buen clima de clase. & 4,5926 & 0,5007 & $100 \%$ \\
\hline $\begin{array}{l}\text { 14. Considero que haber participado activamente en las } \\
\text { actividades del proyecto me ha dado herramientas para } \\
\text { mi futura profesión. }\end{array}$ & 4,5185 & 0,6427 & $93 \%$ \\
\hline
\end{tabular}

Figura 10. Puntuaciones de las capacidades trabajadas

En primer lugar, el alumnado valora de manera positiva ( $96 \%$ y $100 \%$ respectivamente) la relación con el resto del grupo-clase $(M=4,5 ; D T=0,57)$, así como el clima creado en el aula $(M=4,6 ; \mathrm{DT}=0,50)$.

Un 96\% de los estudiantes afirma que la motivación para realizar las actividades que requería la asignatura ha sido muy alta $(M=4,4 ; D T=0,57)$. Lo cual, guarda relación con el hecho de que el porcentaje de alumnado que afirma no haber dedicado todo el tiempo posible a la actividad desciende a un $70 \%(M=3,4 ; D T=1,1)$. Parece que los estudiantes se refieren en ese caso al tiempo fuera del aula, ya que, por otro lado, el 100\% afirman con las puntuaciones "todo y bastante" ( $M=5$; DT=1) haberse esforzado todo lo posible en las acciones.

Por otro lado, el alumnado reconoce haberse esforzado en conocer y comprender a personas que no pertenecen a su contexto $(M=4,7$; $D T=0,56)$ y afirma que la experiencia de ApS vivida les ha servido para estrechar lazos con la comunidad ( $M=4,4$; DT=0,69).

Finalmente, el $100 \%$ alumnado considera con un "bastante" o "todo" ( $M=5$; $\mathrm{DT}=1$ ) que el haber participado en el proyecto les ha hecho crecer como persona. Con el mismo porcentaje se afirma haber comprendido la relación entre servicio y aprendizaje $(M=5 ; D T=0,48)$. 
Tras el análisis por ítems, se pasa a describir los resultados por dimensiones (Figura 11).

\begin{tabular}{llc}
\hline Dimensión analizada & Indicadores & Puntuaciones \\
\hline Compromiso ético & $\begin{array}{l}\text { Responsabilidad } \\
\text { Esfuerzo } \\
\text { Participación } \\
\text { Comprensión } \\
\text { Asistencia } \\
\text { Planificación y gestión del tiempo } \\
\text { Dedicación } \\
\text { Puntualidad }\end{array}$ & 4,75 \\
Auto)Motivación & $\begin{array}{l}\text { Motivación } \\
\text { Implicación }\end{array}$ & 4,14 \\
Capreciación de la diversidad & $\begin{array}{l}\text { Conocimiento y comprensión } \\
\text { Lazos }\end{array}$ & 4,39 \\
Aplicación conocimientos & $\begin{array}{l}\text { Relación grupo-clase } \\
\text { Clima }\end{array}$ & 4,53 \\
\hline
\end{tabular}

Figura 11. Puntuaciones medias por dimensiones

De los constructos analizados, el compromiso ético es el que mayor puntuación media posee, sin embargo, las desviaciones típicas de las medias de los ítems que los componen son también las más altas.

La planificación y gestión del tiempo ha sido la capacidad peor valorada con una $M=4,14$ y una variación en sus medias y las desviaciones típicas alta. Por otro lado, el alumnado valora con un 4,52 de 5 de media la experiencia, como oportunidad para aplicar conocimientos. El alumnado además afirma que con actividades de este tipo la capacidad de relación entre el grupo clase mejora $M=4,54$. Por último, tanto la apreciación de la diversidad, como la motivación hacía la tarea también reciben medias altas.

\section{DisCUSIÓN Y CONCLUSIONES}

El verdadero éxito del sistema educativo consiste en formar ciudadanía capaz de mejorar la sociedad y no sólo su currículum personal. Para mejorar la sociedad el centro educativo debe colaborar activamente con los agentes sociales de la comunidad: ONG, ayuntamiento, entidades sociales y educativas del barrio, fundaciones, etc. Lo cual significa que la metodología didáctica no puede ser la tradicional, centrada en "tomar apuntes" (Bisquerra, 2008, p. 87), sino que debe incluir dinámicas diversas, participativas y en conexión con la comunidad. Esto es lo que se ha intentado lograr a través de la experiencia de ApS descrita.

Se trata de cambiar una cultura de trabajo aislado por una cultura de trabajo en red, y en eso, el ApS proporciona múltiples oportunidades. Oportunidades de aprendizaje positivas, reales y significativas, en las que se asegura el protagonismo del alumnado en la búsqueda de soluciones a problemas complejos y reales que afectan a la comunidad a la que pertenecen y que requieren ser resueltos cooperativamente 
y en equipo (Lucas, 2012). De modo que el alumnado comprende así que la participación en la solución de los problemas de la comunidad se relaciona estrechamente con los aprendizajes adquiridos en el aula, y consiguientemente aumenta su motivación, y se favorece su desarrollo personal, emocional y social.

Por otro lado, el desarrollo profesional docente requiere de espacios y tiempos, que provoquen la reflexión de la práctica docente. En este sentido, se aceptan las hipótesis de las que se partía al inicio, habiendo logrado la implicación del alumnado en el desarrollo de sus competencias profesionales y personales. Además, parece que experiencias como la descrita contribuyen a trabajar valores y capacidades transversales en el grupo-clase. En este caso, el ApS ha demostrado ser una excelente metodología que permite poner en práctica lo aprendido, enseña unos valores, conciencia sobre una realidad y aumentar el capital social capacitando al alumnado para ser protagonistas de su propio desarrollo (González Alcocer, 2013).

Algunas investigaciones (Billig, 2002; Eyler, Giles, Stenson y Gray, 2001 y Eyler y Giles, 1999) categorizan los resultados de la aplicación del ApS en cuatro apartados: académicos, personales, sociales y de ciudadanía. Nuestro estudio se ha centrado en especialmente en los apartados personal y social y tras el análisis de los resultados, puede afirmarse que ambos han obtenido puntuaciones altas.

La experiencia de ApS no solo ha servido para combinar la reflexión con la práctica, sino que se ha convertido en una excelente oportunidad-valorada por el alumnado- en la que ir construyendo la propia identidad profesional. Las condiciones de trabajo han supuesto más motivadoras, lo cual se ha traducido en un buen clima de trabajo y coincide con las investigaciones de Mayor (2009). En este sentido no se han enseñado capacidades o competencias, sino que se han empleado, puesto en marcha y después analizado (Zabalza, 2012).

En conclusión, a partir del objetivo planteado y tras el análisis de los resultados obtenidos, puede concluirse que el alumnado valora de manera muy positiva la actividad de ApS y sus beneficios para el desarrollo de una serie de capacidades personales y profesionales.

Por último, el presente artículo pretende realizar un pequeño aporte en dos sentidos. En primer lugar, como muestra de la excelente respuesta y opinión del alumnado ante experiencias de ApS. Y, en segundo lugar, como una propuesta de evaluación de experiencias de este tipo, campo no tan desarrollado. Sin embargo, en este sentido y de cara a futuras investigaciones, cabe señalar como limitaciones: el tamaño de la muestra o el interés de contar en futuras ocasiones con un grupo control con el que contrastar los resultados.

\section{ReFERENCIAS BIBLIOGRÁFICAS}

Alonso, C. (2004). La Creatividad en la universidad: Reflexiones para la profundización en el ámbito de la docencia. Creatividad y Sociedad, 6, 47-57.

Alonso, I., Arandia, M., Martínez, I., Martínez, B. y Gezuraga, M. (2013). El aprendizaje-Servicio en la innovación universitaria. Una experiencia realizada en la formación de educadoras y educadores sociales. Revista Internacional de Educación para la Justicia Socal (RIEJS), 2(2), 195-216. 
Ander-Egg, E. (2000). Introducción a la planificación. Buenos Aires: Humanitas.

Arandia, M. y Fernández, I. (2012). ¿Es posible un curriculum más allá de las asignaturas? Diseño y práctica del grado de educación social en la Universidad del País Vasco. REDU: Revista de Docencia Universitaria, 10(3), 99-123.

Batle, R. (2010). Aprendizaje-servicio y entidades sociales. Cuadernos de Pedagogía, 192, 66-68.

Billing, S. y Waterman, A. (2014). Studying Service-learning. Innovation in education research methodology. New York: Lawrence Erlbaum Associates.

Bisquerra, R. (2008). Educación para la ciudadanía y convivencia. El enfoque de la educación emocional. Madrid: Wolters Kluwer.

Bologna Working Group (2005). A Framework for Qualifications of the European Higher Education Area. Bologna Working. Copenhagen: Danish Ministry of Science, Technology and Innovation.

Bourn, D. y Brown, K. (2011). Young People and International Development. (Research Paper, $n^{\circ}$ 2). London: Development Education Research Centre.

Bringle, R. y Hatcher, J. (2009). Innovative practices in service-learning and curricular engagement. New directions for Higher Education, 147, 37-46. DOI: http://doi. org/10.1002/he.356.

Brown, E. J. (2013). Transformative Learning through Development Education NGOs: A Comparative Study of Britian and Spain. Tesis doctoral inédita. University of Nottingham.

Campbell, C. y Rozenyai, C. (2002). Quality assurance and the development of course programming. Papers on Higher Education Regional University Naturals on Governance and Management of Higher Education in South East Europe Bucharest. Paris: UNESCO.

Carrica-Ochoa, S. (2012). Aprendizaje y Servicio: un buen medio de Educación para el Desarrollo. En L. Rubio, E. Prats, y L. Gómez (coords.) Universidad y sociedad. Experiencias de aprendizaje servicio en la universidad (pp. 316-321). Barcelona: Universitat de Barcelona (Institut de Ciències de l'Educació).

Castilla, F. (2011). Calidad docente en el ámbito universitario: un estudio comparativo de las universidades andaluzas. EDUCADE, 2, 157-172.

CONCORD (2011). Development needs citizens: The place of Development Education \& Awareness Raising in the development discourse. Brussels: DEEEP.

Debowsky, S. (2012). The New Academic: A Strategic Handbook. England: McGraw Hill.

Gil-Gómez, J., Moliner-García, O., Chiva-Bartoll, O. y García-López, R. (2014). Una experiencia de Aprendizaje-Servicio en futuros docentes: desarrollo de la competencia social y ciudadana. Revista Complutense de Educación, 27(1), 53-73.

Glennie, A., Straw, W. y Wild, L. (2012). Understanding public attitudes to aid and development. London: Institute for Public Policy Research \& Overseas Development Institute.

Goleman, D. (1998). La práctica de la inteligencia emocional. Barcelona: Kairós.

González Alcocer, J. R. (2013). Desarrollo Comunitario y educación. Recuperado de: http://www.colectivoescuelaabierta.org/escuelaV_2013/Desarrollo\%20cumunitario.pdf. 
Gewerc, A. (2011). Identidades docentes en contextos turbulentos. Espacios, tiempos y afectos. En C. Monereo y J. I. Pozo, La identidad en Psicología de la educación (pp. 189-212). Madrid: Narcea.

Hérbert, A. y Hauf, P. (2015). Student learning through service-learning: effects on academic development, civic responsibility, interpersonal skills and practical skills. Active learning in Higher Education, 16(1), 37-49. DOI: http://doi. org/10.1177/1469787415573357.

Hogg, M. (2011). Do we need a deeper, more complex conversation with the public about global issues? London: Think Global.

Lantieri, L. (2009). Inteligencia emocional infantil y juvenil. Madrid: Aguilar.

Lucas, S. (2012). Aprendizaje-Servicio como propuesta de integración curricular del Voluntariado en la Responsabilidad Social Universitaria. Actas de Jornadas sobre Responsabilidad Social 2009-2010-2011 (p. 27). Valladolid: Universidad de VaIladolid; Caja de Burgos.

Martín-Gutiérrez, A., Conde-Jiménez, J. y Mayor-Ruiz, C. (2014). La identidad profesional docente del profesorado novel universitario. REDU - Revista de Docencia Universitaria, 12(4),141-160.

Martínez Odría, A, (2005). Service-Learning o Aprendizaje-Servicio: Una propuesta de incorporación curricular del voluntariado. Tesis Doctoral. Universidad de Navarra.

Martínez, B., Martínez, I., Alonso, I. y Gezuraga, M. (2013). El A-S, una oportunidad para avanzar en la innovación educativa dentro de la Universidad del País Vasco. Tendencias Pedagógicas, 21, 99-117.

Martínez, M. (2010). Aprendizaje Servicio y Responsabilidad Social de las Universidades. Barcelona: Octaedro.

Martínez-Vivot, M. y Folgueiras, P. (2015). Evaluación participativa, Aprendizaje-Servicio y Universidad. Revista de currículum y formación del profesorado, 19(1), 128-143.

Mayor, C. (2009). El desafío de los profesores principiantes universitarios. En C. Marcelo (coord.), Profesores principiantes e inserción a la docencia (pp. 177-211). Barcelona: Octaedro.

Mayor, C. (2011). La evaluación del desarrollo profesional basado en la supervisión: El caso de los profesores principiantes. En C. Marcelo (coord.), Evaluación del Desarrollo profesional docente. Barcelona: Davinci.

Onu, V. C., Eskay, M. K., Obiyo, N. O., Igbo, J. N. y Ezeanwu, A. B. (2012). Innovation for Transformation in Nigeria University Education: Implication for the Production of Critical and Creative Thinkers. US-China Education Review, A2, 229-236.

Palomares, A. (2011). El modelo docente universitario y el uso de nuevas metodologías en la enseñanza, aprendizaje y evaluación. Revista de Educación, 355, 591-604.

Puig, J. M., Batlle, R., Bosch, C. y Palos, J. (2007). Aprendizaje y Servicio. Educar para la ciudadanía. Madrid: Ministerio de Educación y Ciencia.

Siegel, D. J. (2011). Mindsight. La nueva ciencia de la transformación personal. Barcelona: Paidós. 
Valcárcel, M. (coord.) (2003). La Preparación del Profesorado Universitario Español para la Convergencia Europea en Educación Superior. PROYECTO EA2003- 0040. Recuperado de: http://campus.usal.es/web-usal/Novedades/noticias/bolonia/ informe_final.pdf (Consultado 21-10-2013).

Zabalza, M. A. (2011). Formación del profesorado universitario: mejorar a los docentes para mejorar la docencia. Educação, Santa Maria, 36(3), 397-424.

Zabalza, M. A. (2012). Las competencias en la formación del profesorado: de la teoría a las propuestas práctica. Tendencias Pedagógicas, 20, 5-32. 